\title{
Raspberry alleviates obesity-induced inflammation and insulin resistance in skeletal muscle through activation of AMP- activated protein kinase (AMPK) a1
}

\author{
Liang Zhao $\mathbb{0}^{1}$, Tiande Zou ${ }^{1,2}$, Noe Alberto Gomez ${ }^{1}$, Bo Wang ${ }^{1}$, Mei-Jun Zhu ${ }^{3}$ and Min Du ${ }^{1,4}$
}

\begin{abstract}
OBJECTIVE: Through dynamic means, etiological factors, including chronic inflammation and insulin resistance have the potential to perpetuate metabolic incidences such as type 2 diabetes and obesity. Abatement of such syndromes can be achieved by complex mechanisms initiated through bioactive compounds such as polyphenols derived from fruits. Using a whole-fruit approach, the effects of dietary red raspberry, which is rich in polyphenols, on inflammatory responses and insulin resistance in the skeletal muscles of Mus musculus were studied along with the potential role of AMP-activated protein kinase (AMPK) to act as a key mediator.
\end{abstract}

SUBJECTS: Wild-type (WT) mice and mice deficient in the catalytic subunit (a1) of AMPK (AMPKa ${ }^{-1-}$ ) were fed with a high-fat diet (HFD) or HFD supplemented with raspberry (5\% dry weight) for 10 weeks. Factors involved in inflammatory responses, insulin signaling transduction, and mitochondrial biogenesis were evaluated.

RESULTS: Dietary raspberry reduced ectopic lipid storage, alleviated inflammation responses, improved whole-body insulin sensitivity, and promoted mitochondrial biogenesis in the skeletal muscle of WT mice, but not AMPKa1 ${ }^{-1-}$ mice.

CONCLUSIONS: AMPKa1 is an important mediator for the beneficial effects of raspberry through alleviating inflammatory responses and sensitizing insulin signaling in skeletal muscle of HFD-fed mice.

\section{INTRODUCTION}

Red raspberry is widely recognized for its high levels of vitamin $\mathrm{C}$ and bioactive polyphenols, including ellagitannins and anthocyanins, which have strong antioxidant capacities $^{1}$. Several animal studies have shown that supplementation of raspberry extracts exhibited beneficial effects for the prevention of obesity, inflammation and

\footnotetext{
Correspondence: Min Du (min.du@wsu.edu)

'Department of Animal Sciences, Nutrigenomics and Growth Biology laboratory, Washington State University, Pullman, WA 99164, USA ${ }^{2}$ Jiangxi Province Key Laboratory of Animal Nutrition, College of Animal Science and Technology, Jiangxi Agricultural University, Nanchang, Jiangxi 330045, China

Full list of author information is available at the end of the article.
}

other metabolic diseases ${ }^{2,}{ }^{3}$. However, the impacts of dietary raspberry fruit on skeletal muscle insulin resistance and the underlying mechanisms remain largely unexplored.

Obesity induces ectopic lipid accumulation and desensitizes insulin signaling in skeletal muscle, thus resulting in systematic insulin resistance and type 2 diabetes ${ }^{4}$. AMP-activated protein kinase (AMPK) is a key sensor of energy status in skeletal muscle through the control of glucose and fatty acid metabolism ${ }^{5}$. The structure of AMPK has been described as a heterotrimeric complex comprised of the catalytic $\alpha$-subunit and the regulatory $\beta$ and $\gamma$ - subunits ${ }^{6}$. Activation of AMPK prevents obesity

\section{(c) The Author(s) 2018}

(c) (i) Open Access This article is licensed under a Creative Commons Attribution 4.0 International License, which permits use, sharing, adaptation, distribution and reproduction in any medium or format, as long as you give appropriate credit to the original author(s) and the source, provide a link to the Creative Commons license, and indicate if changes were made. The images or other third party material in this article are included in the article's Creative Commons license, unless indicated otherwise in a credit line to the material. If material is not included in the article's Creative Commons license and your intended use is not permitted by statutory regulation or exceeds the permitted use, you will need to obtain permission directly from the copyright holder. To view a copy of this license, visit http://creativecommons.org/licenses/by/4.0/. 
and associated metabolic diseases through the promotion of glucose utilization, fatty acid oxidation, and mitochondrial biogenesis in skeletal muscle ${ }^{6}$. Dietary polyphenols, such as resveratrol, are strong activators of AMPK, which can then promote the browning of white adipose and subsequently alleviate obesity ${ }^{7}$. Due to the high levels of polyphenols found in the red raspberry, it is postulated that AMPK plays an essential role in mediating the beneficial effects of red raspberry on metabolic health.

The catalytic subunit of AMPK has 2 isoforms ( $\alpha 1$ and $\alpha 2)$. Although there is a compensatory mechanism between these two isoforms, their expression shows tissuespecific patterns ${ }^{8,9}$ with differential metabolic functions ${ }^{10 \text {, }}$ ${ }^{11}$. The isoform $\alpha 2$ of AMPK is indispensable for increased glucose uptake by skeletal muscle induced by 5aminoimidazole-4-carboxamide ribonucleotide (AICAR) and hypoxia ${ }^{5,12,13}$. Meanwhile, the AMPK $\alpha 1$ isoform can be activated during skeletal muscle contraction ${ }^{14}$ and at low caffeine concentrations ${ }^{10}$. Indeed, AMPK $\alpha 1$ also plays an essential role in myogenin expression and myogenesis $^{15}$. Previous studies in our lab have shown the dominant expression of AMPK $\alpha 1$ in satellite cells, which when deleted, impeded muscle regeneration after injury ${ }^{15}$. Deletion of AMPK $\alpha 1$ in macrophages during the transition from a proinflammatory (M1) to an antiinflammatory (M2) phenotype impairs the resolution of inflammation and muscle regeneration after injury ${ }^{16}$. Altogether, these studies suggested that AMPK $\alpha 1$ could mediate the alleviation of insulin resistance and metabolic syndromes in skeletal muscle of obese mice consuming raspberry. Thus, we explored the influence of red raspberry on insulin sensitivity and inflammatory responses in skeletal muscles, along with the potential role of AMPK $\alpha 1$ to act as a key mediator.

\section{MATERIALS AND METHODS}

Animal and experimental design

$\mathrm{R} 26^{\mathrm{Cre}} / \mathrm{AMPK \alpha} 1^{\mathrm{f} / \mathrm{fl}}$ mice were generated through the cross-breeding of $A M P K \alpha 1^{\mathrm{fl} / \mathrm{fl}}$ mice (Stock No: 014141, Jackson Lab, Bar Harbor, Maine) with tamoxifeninducible R26-Cre mice (Stock No: 004847, Jackson Lab, Bar Harbor, Maine) at Washington State University. To induce the AMPK $\alpha 1$ knockout (AMPK $\alpha 1^{-1-}$ ), 2-monthold male $\mathrm{R} 26^{\mathrm{Cre}} / \mathrm{AMPK} \alpha 1^{\mathrm{f} / \mathrm{fl}}$ mice were intraperitoneally injected with tamoxifen $(75 \mathrm{mg} / \mathrm{kg}$ body weight) for 4 continuous days ${ }^{17}$. AMPK $\alpha 1^{\mathrm{f} / / \mathrm{fl}}$ mice treated with tamoxifen were used as controls (Wild-type, WT). To minimize possible confounding changes, dietary treatments started 3 days after the last tamoxifen injection ${ }^{15}$. All experimental procedures of animal use were performed according to the guidelines of National Institutes of Health and approved by the Animal Use and Care Committee of Washington State University (Permit No. 04719).
Twelve wild-type and AMPK $\alpha 1^{-/-}$mice, respectively, were randomly separated into two sub-groups and fed either a high-fat diet (HFD; 60\% energy from fat, D12492; Research Diets, New Brunswick, NJ, USA) or a HFD diet supplemented with freeze-dried raspberry (5\% of dry feed weight, red raspberry powder). The concentration of the raspberry supplementation was determined by preliminary studies in our lab ${ }^{18}$. Raspberry powder was prepared as previously described, which contains polyphenols at $\sim 11 \mathrm{~g}$ gallic acid equivalent (GAE) $/ \mathrm{kg}$ of dry weight, $4.24 \pm 0.12 \%$ protein, $1.91 \pm 0.03 \%$ fat, $0.81 \pm 0.02 \%$ ash, $16.14 \pm 0.45 \%$ moisture, and the remaining to be mainly carbohydrates $^{19}$. Mice were housed in a temperaturecontrolled environment $\left(23 \pm 2{ }^{\circ} \mathrm{C}\right.$, alternating 12-h light/ dark cycle) with ad libitum access to food and water. Feed intake and body weights were monitored weekly until the mice were killed 10 weeks later. Samples of blood, the Gastrocnemius muscle (GA), and the Tibialis anterior muscle (TA) were rapidly isolated. TA were fixed in $4 \%$ paraformaldehyde for sectioning and staining, and GA were rapidly frozen in liquid nitrogen and stored at $-80^{\circ}$ $\mathrm{C}$ until further analyses.

\section{Histochemical analyses}

Paraffin-embedded TA muscle sections (5- $\mu \mathrm{m}$ thick) were rehydrated through a series of incubations in xylene and ethanol solutions, and then used for Masson trichrome staining ${ }^{20}$. At least four fields per section and four sections per sample were randomly selected for quantification of fat area and collagen area using the Image J 1.46r software (National Institutes of Health). The average data per biological sample were used for calculations.

\section{Total triacylglycerol analyses}

As previously described, total triacylglycerol determination was performed using the Folch method ${ }^{20,}{ }^{21}$. The frozen GA muscle was powdered under liquid nitrogen and a $30 \mathrm{mg}$ sample was weighed. After adding $0.75 \mathrm{ml}$ of chloroform-methanol 2:1 (v/v), the samples were left at $4^{\circ}$ $\mathrm{C}$ for $48 \mathrm{~h}$. Then, $187.5 \mu \mathrm{l} 0.9 \% \mathrm{NaCl}$ was added and the mixture was kept at room temperature overnight and then centrifuged at $10,000 \times g$ for $5 \mathrm{~min}$ at $4{ }^{\circ} \mathrm{C}$. The lower phase $(20 \mu \mathrm{l})$ was transferred into a fresh tube and evaporated until dry for $1 \mathrm{~h}$ under the hood. Total triacylglycerols were measured using a kit from Sigma following the manufacturer's instructions (cat. no. TR0100). The results were displayed by dividing the total triacylglycerol content by the initial muscle powder weight.

\section{Quantitative real-time PCR (qRT-PCR) analyses}

Total RNA was isolated using TRIzol reagent (Sigma, Saint Louis, MO, USA), followed by reverse-transcription to cDNA using the iScriptTM cDNA Synthesis Kit (BioRad, Hercules, CA, USA). The mRNA levels were 


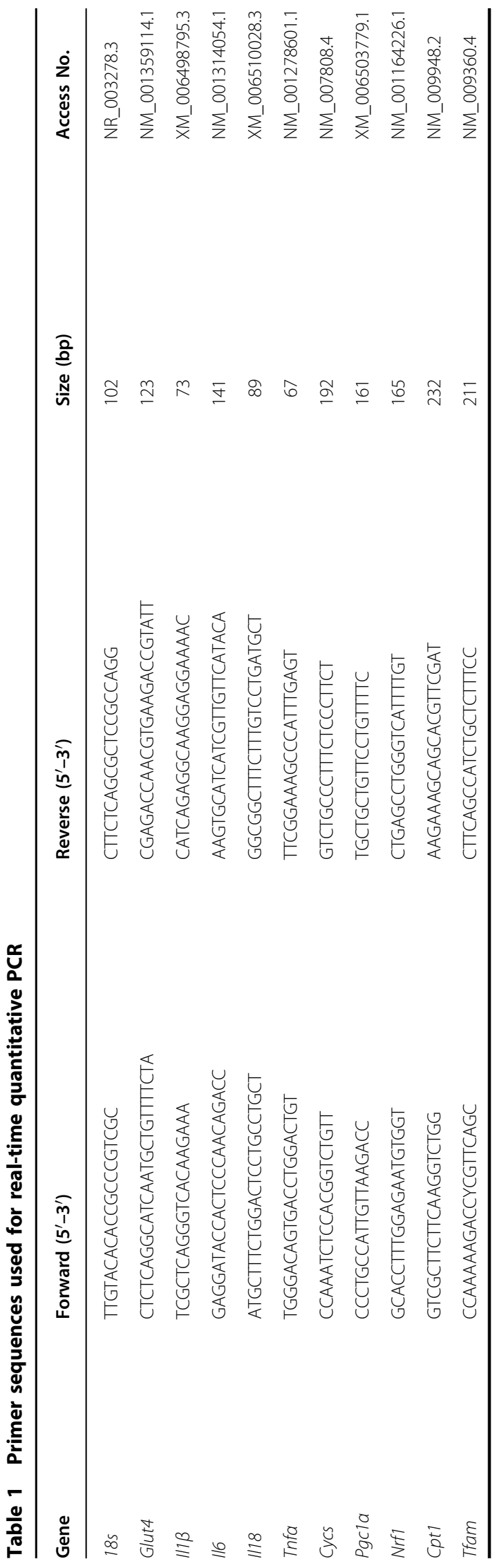

measured by qRT-PCR carried out by the CFX RT-PCR detection system (Bio-Rad). After normalization to $18 \mathrm{~s}$ rRNA content, relative mRNA expression was determined using the method of $2^{-\Delta \Delta \mathrm{Ct} 22}$. Table 1 shows the primer sequences.

\section{Immunoblotting analyses}

Immunoblotting analyses were performed as previously described using the Odyssey Infrared Image System (LICOR Biosciences, Lincoln, NE, USA) ${ }^{15}$. Band densities of target proteins were normalized to $\beta$-tubulin content. The following antibodies were purchased from Cell Signaling (Danver, MA, USA): AMPK $\alpha$ (no.2532), phosphoAMPK $\alpha$ at Thr172 (no. 2535), protein kinase B (AKT, no.9272), phospho-AKT at Ser473 (no. 9271), protein kinase C (PKC $\theta$, no.13643), phospho-PKC $\theta$ at Thr538 (no.9377), nuclear factor $\mathrm{\kappa B}(\mathrm{NF \kappa B})$ subunit $\mathrm{p} 65$ (no.8242), phospho-p65 at Ser536 (no.3033), c-Jun Nterminal kinases (JNK, no. 9252), phospho-JNK at Thr183/Tyr185 (no.9251) and cytochrome C (cyt C, no. 4280). IRDye 800CW goat anti-rabbit (no. 926-32211) and IRDye 680 goat anti-mouse (no. 926-68070) secondary antibodies were purchased from LI-COR Biosciences (Lincoln, NE, USA). For use, primary antibodies were diluted 1: 1000 using 1× TBST buffer (137 mM Sodium Chloride, $20 \mathrm{mM}$ Tris, $0.1 \%$ Tween-20, pH 7.6) with 5\% BSA (Bovine Serum Albumin) and secondary antibodies were diluted 1: 10,000 using TBST buffer.

\section{Statistical analyses}

Within each genotype, the data were analyzed using unpaired two-tailed Student's $t$ test using SAS 9.0 (SAS Institute Inc., Cary, NC, USA). All the data were found normally distributed. Results are expressed as mean \pm s.d. A significant difference was considered as $P<0.05$.

\section{RESULTS}

\section{Raspberry supplementation activated AMPKa1}

The content of total AMPK $\alpha$ in skeletal muscles was lower in $A M P K \alpha 1^{-1-}$ mice (Fig. 1a), which is consistent with successful AMPK $\alpha 1$ knockout induced by tamoxifen. Raspberry supplementation increased the level of $\mathrm{p}$ AMPK $\alpha$ and the ratio of $\mathrm{p}-/ \mathrm{t}$-AMPK in WT mice, while no difference was found in AMPK $\alpha 1^{-1-}$ mice with/ without raspberry (Fig. 1b). The lack of difference in AMPK phosphorylation and ratio of $\mathrm{p}$-/t-AMPK in the absence of AMPK $\alpha 1$ suggests that raspberry supplementation did not activate AMPK $\alpha 2$.

\section{Raspberry supplementation reduced lipid accumulation in} skeletal muscles in an AMPKa1-dependent manner

As described previously in our lab, there was no significant difference of average weekly food intake between groups $(p>0.05)$ and dietary raspberry reduced the body 
A

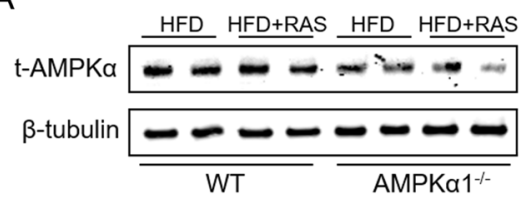

B

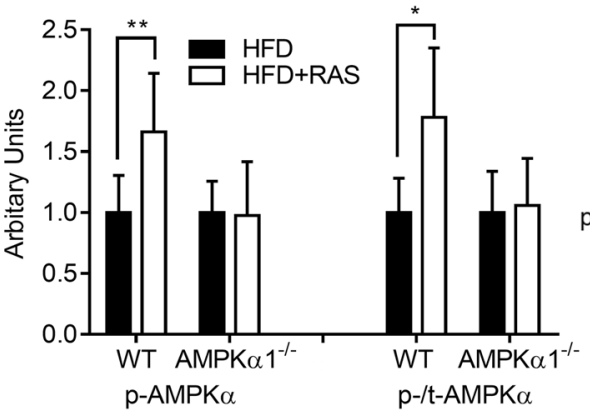

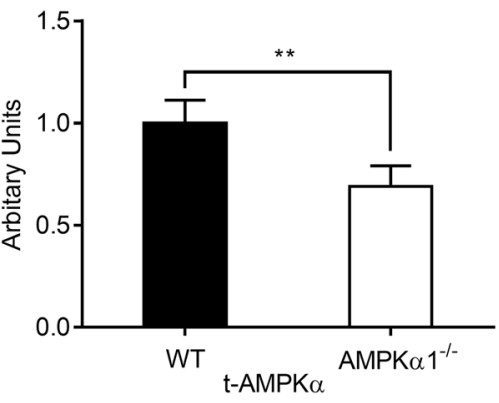

HFD HFD+RAS HFD HFD+RAS

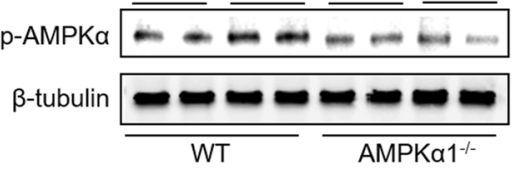

Fig. 1 Contents of total AMPKa (a) and phospho-AMPKa (b) in the Gastrocnemius muscle of wild-type (WT) and AMPKa1 knockout mice with/without raspberry supplementation. ( ${ }^{*} P<0.01,{ }^{*} P<0.05, \mathrm{HFD}+$ RAS vs. HFD only for each genotype as determined by unpaired two-tailed Student's $t$ test; mean \pm s.d.; $n=6$ ). t total content, $p$ phosphorylated form

weight of wide type mice but not that of the AMPK $\alpha 1^{-1-}$ mice $(p<0.01)^{23}$. The TA and GA muscle weights were not altered through raspberry supplementation, nor by AMPK $\alpha 1$ deficiency (Fig. 2a, b).

Intramuscular lipid accumulation contributes to obesityinduced insulin resistance by activating stress-responsive serine kinases and then impeding the activity of downstream insulin signaling molecules such as $\mathrm{AKT}^{24,25}$. The triacylglycerol content in the GA muscle was elevated due to the HFD, but partially prevented by dietary raspberry in WT mice. For the AMPK $\alpha 1 \mathrm{KO}$ mice, no difference was found between HFD and HFD + RAS groups (Fig. 2c), supporting the mediatory role of AMPK $\alpha 1$.

Masson trichrome staining shows the areas of muscle cells in red, collagen in blue, and adipocytes as colorless. More intramuscular adipocytes in TA muscle were observed in the HFD group compared to the HFD + RAS group of WT mice as shown in Fig. 2d. The areas of fat (Fig. 2e) and collagen (Fig. 2f) in muscle sections were quantified. Fat area was much smaller $(P<0.01)$ in the HFD + RAS group compared to the HFD group of WT mice, consistent with the lower levels of triacylglycerols in the HFD + RAS group of WT mice as shown in Fig. 2c. Raspberry supplementation also decreased the presence of connective tissues in WT mice. A tendency for a decrease in collagen area was seen in the HFD + RAS group of WT mice $(P<0.10)$. For AMPK $\alpha 1^{-1-}$ mice, no significant difference was exhibited for either fat and collagen areas.

These data suggest that raspberry supplementation reduced lipid accumulation in skeletal muscle of mice challenged with a HFD diet, a process mediated by AMPK $\alpha 1$.

\section{Raspberry supplementation decreased the inflammatory} response in an AMPKa1-dependent manner

Ectopic lipid accumulation in peripheral tissues frequently leads to chronic inflammation. Raspberry intake attenuated HFD-stimulated expression of Tnfo, Il1 $\beta$, Il6, and Ill8 in WT mice (Fig. 3a). However, this beneficial role of raspberry supplementation was not present in $\mathrm{AMPK} \alpha 1^{-1-}$ mice.

Inflammatory responses are mediated by the activation of NF-kB (nuclear factor kappa B) and JNK/MAPK pathways $^{26-28}$. Protein p65 is a key component of the NF- $\mathrm{KB}$ pathway with obesity up-regulating its phosphorylation ${ }^{20}$. Although the total contents of p65 did not change, a much lower phosphorylation level of p65 and a low phospho to total ratio of p65 (p-p65/t-p65) were detected in the HFD + RAS group of WT mice (Fig. 3b). In AMPK $\alpha 1^{-1-}$ mice, raspberry supplementation did not reduce the phosphorylation level of p65. In addition, raspberry supplementation also decreased the phosphorylation level of JNK in WT mice (Fig. 3c). Although the total level of JNK and the ratio of $\mathrm{p}-/ \mathrm{t}$-JNK showed a decreasing tendency, changes were not significant. However, these benefits disappeared in AMPK $\alpha 1^{-l-}$ mice, showing the mediatory role of AMPK $\alpha 1$.

\section{Raspberry improved insulin sensitivity in an AMPKa1- dependent manner}

Previous studies in our laboratory have reported that raspberry supplementation increased glucose tolerance, and decreased lipids and insulin levels in the serum of WT mice but not in AMPK $\alpha 1^{-1-}$ mice, which reflected 

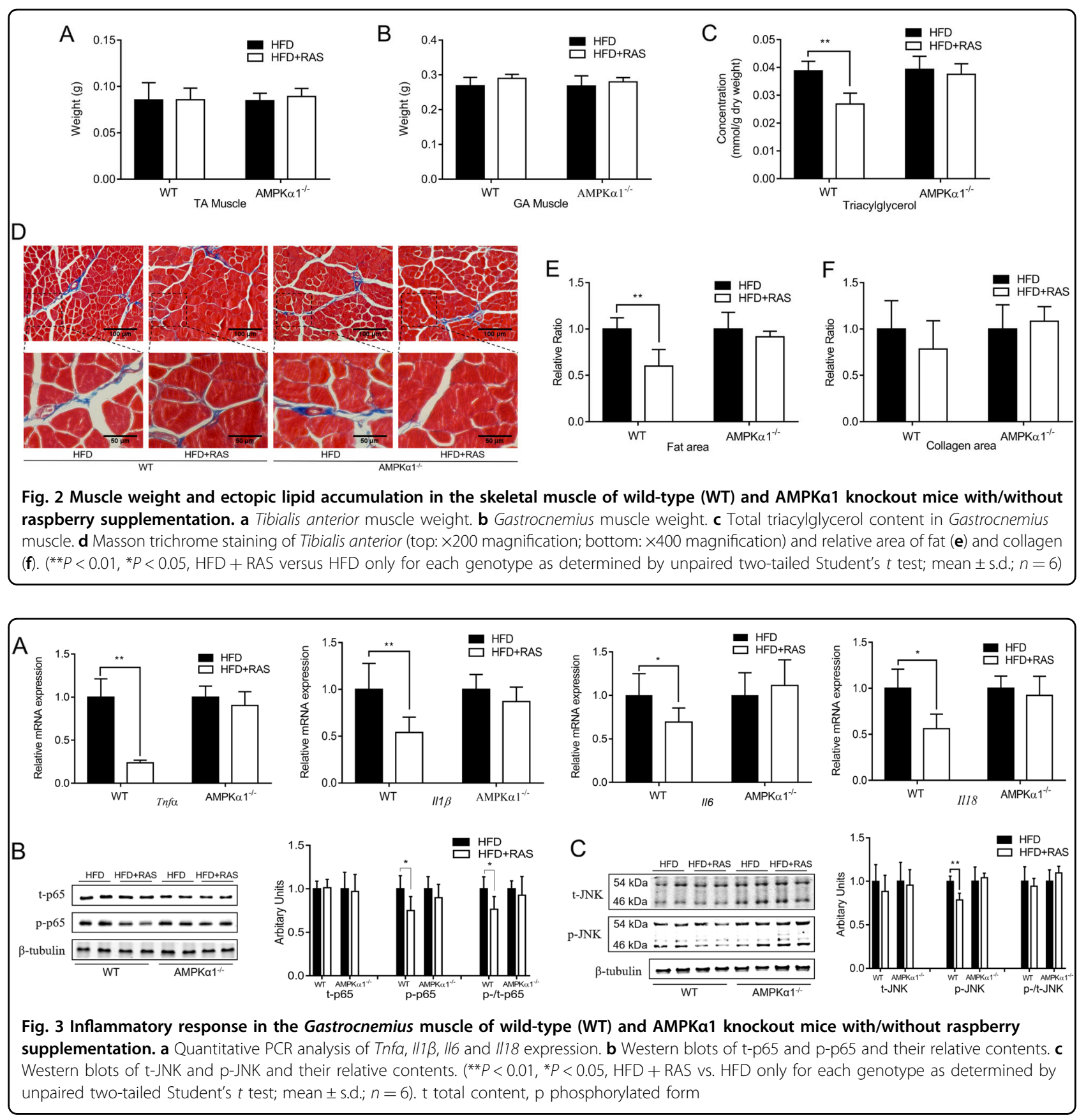

improved insulin sensitivity by raspberry supplementation through regulating AMPK $\alpha 1^{23}$.

Glucose transporter 4 (GLUT4) is indispensable for whole-body glucose homeostasis and its deficiency leads to insulin resistance and ectopic lipid accumulation ${ }^{29,} 30$. Consistently raspberry supplementations increased Glut4 mRNA and protein contents in WT mice but not in AMPK $\alpha 1^{-1-}$ mice (Fig. 4a, b).

Because increased lipid accumulation and inflammation are correlated with insulin resistance, insulin signaling pathways were further analyzed. In WT mice, the contents of PKC $\theta$ and its phosphorylation were downregulated by $19.5 \%(p<0.1)$ and $27.5 \%(p<0.01)$ in raspberry supplemented group, respectively. In the absence of AMPK 1 , however, these differences disappeared (Fig. 4c).

Although the total level of AKT was not different, its phosphorylation was higher $(P<0.01)$ in RAS supplemented WT mice when compared to those fed only HFD (Fig. 4c). Consequently, the HFD + RAS group of WT mice had a significantly higher $\mathrm{p}-/ \mathrm{t}$-AKT ratio $(P<0.05)$. Ablation of AMPK $\alpha 1$ abolished these changes induced by 

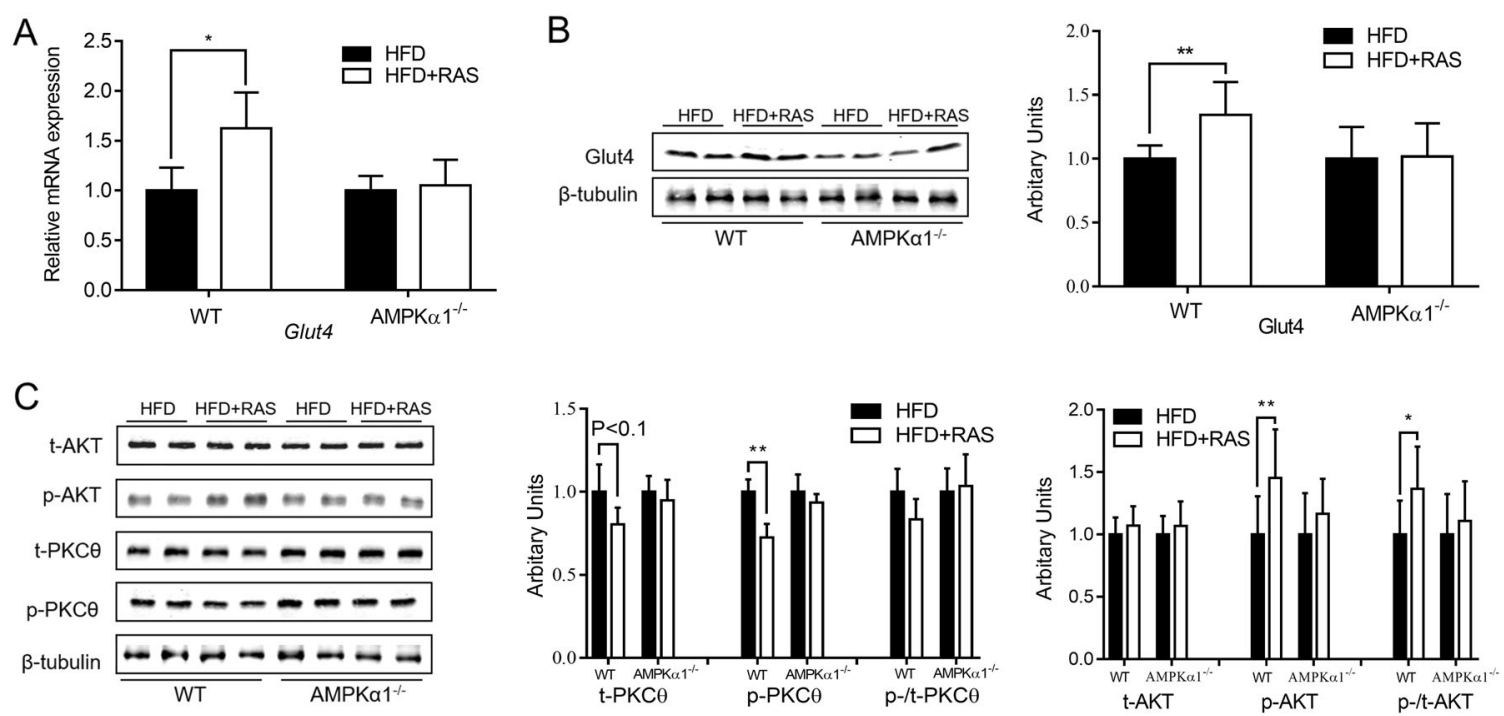

Fig. 4 Expression of Glucose transport 4 (GLUT4) and relative contents of insulin signaling proteins in the Gastrocnemius muscle of wildtype (WT) and AMPKa1 knockout mice with/without raspberry supplementation. a Quantitative PCR analysis of Glut4 expression. $\mathbf{b}$ Western blots of Glut4 and their relative contents. c Western blots of insulin signaling proteins (t-AKT, p-AKT, t-PKC $\theta$ and p-PKC $\theta$ ) and their relative contents. $\left({ }^{* *} P<0.01,{ }^{*} P<0.05, \mathrm{HFD}+\right.$ RAS versus HFD only for each genotype as determined by unpaired two-tailed Student's $t$ test; mean $\left.\pm s . d . ; n=6\right), t$ total content, $\mathrm{p}$ phosphorylated form

raspberry supplementation. Therefore, AMPK $\alpha 1$ is required for the beneficial effects of raspberry on insulin signaling in skeletal muscle of mice under the challenge of HFD.

\section{Raspberry promoted mitochondrial biogenesis in an AMPKa1-dependent manner}

The mitochondria play an indispensable role in cellular energy metabolism while its dysfunction in skeletal muscle is associated with decreased insulin sensitivity and the development of type 2 diabetes $^{31}$. Raspberry supplementation increased the protein level of cytochrome $\mathrm{C}$ (Cyt $C)$ in skeletal muscle $(p<0.01)$, suggesting increased contents of mitochondria (Fig. 5a). Meanwhile, the mRNA expression levels for Pgc1 $\alpha$, Nrf1, and Cpt1 were upregulated in the HFD + RAS group of WT mice (Fig. 5b). However, in AMPK $\alpha 1^{-/-}$mice, no such differences were observed. The mRNA expression of Cycs and Tfam did not differ between WT and AMPK $\alpha 1^{-1-}$ groups. In summary, increased mitochondrial biogenesis could be responsible for the reduced lipid accumulation elicited by raspberry supplementation in WT mice challenged with HFD in an AMPK $\alpha 1$-dependent manner.

\section{DISCUSSION}

Obesity and associated chronic inflammations induce a state of insulin resistance in adipose tissue, skeletal muscles, and the liver, which is indispensable for the development of type 2 diabetes $^{32}$. Numerous pharmaceutical approaches aimed at preventing obesity and inflammation have shown positive results, but with various side effects and risks ${ }^{33}$. Nutritional interventions have the advantage of being natural and safe, providing a more suitable alternative for long-term therapy. Raspberries contain high amounts of polyphenols and other bioactive compounds and have been shown to have beneficial effects in treating obesity and metabolic diseases ${ }^{3,}$ 34. However, the effects of raspberry in insulin resistance of skeletal muscle and the mediatory role of AMPK have not been examined.

Obesity induces ectopic lipid storage and inflammatory response, accompanied by the secretion of proinflammatory cytokines such as TNF $\alpha$, IL $1 \beta$ and IL6 ${ }^{31}$. The bioactive polyphenols in red raspberry occur primarily as ellagitannins and anthocyanins, which have antiinflammatory effects ${ }^{1,35}$. In the current study, raspberry supplementation promoted insulin signaling, reduced lipid accumulation, and alleviated the inflammatory response in skeletal muscle. These benefits disappeared in AMPK $\alpha 1$ knockout mice, which showed the indispensable role of AMPK $\alpha 1$ in mediating the beneficial effects of dietary raspberry. Increased mitochondrial biogenesis in WT mice due to raspberry consumption could be a causative reason for these beneficial effects. Following AMPK $\alpha 1$ knockout, raspberry-stimulated mitochondrial biogenesis disappeared, supporting the mediatory role of AMPK $\alpha 1$.

AMPK is a promising drug target for preventing and treating obesity and associated metabolic disease ${ }^{36}$. Increasing the activity of AMPK in skeletal muscles is 


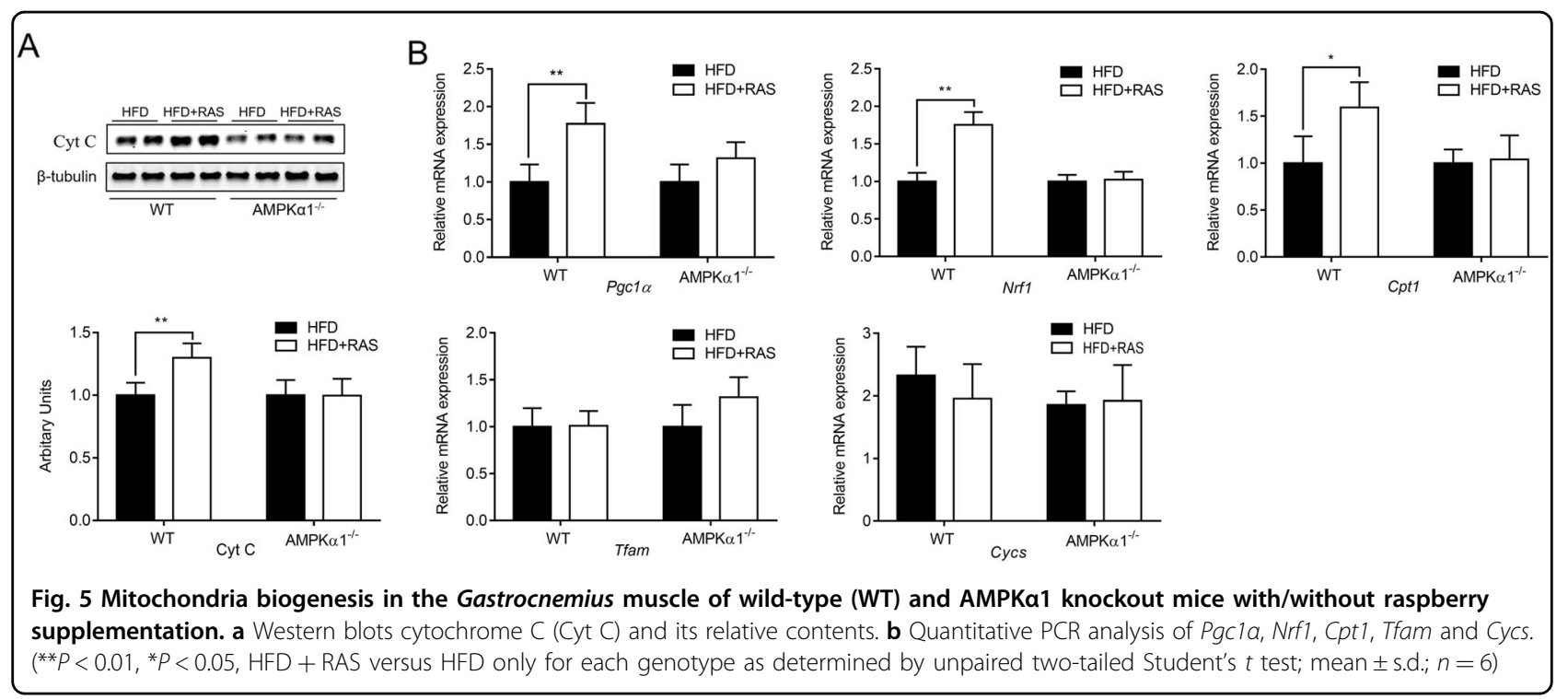

associated with enhanced mitochondrial biogenesis and lipid oxidation ${ }^{7}$. The two catalytic $\alpha$ isoforms ( $\alpha 1$ and $\left.\alpha 2\right)$ of AMPK have different tissue expression patterns. AMPK $\alpha 1$ is widely expressed in all tissues while predominately in brain and adipose tissues, whereas both $\alpha 1$ and $\alpha 2$ isoforms are expressed in skeletal muscles and the heart ${ }^{37}$. Their difference in subcellular localization and substrate specificity also suggest their differential roles in the regulation of metabolic processes ${ }^{8,9}$. AMPK is normally activated in response to an energy-depleting state ${ }^{17}$. Due to allosteric activation by AMP and covalent activation by upstream kinases, AMPK $\alpha 2$ activation is more dependent on AMP and energy depletion than the $\alpha 1$ isoform $^{8,}{ }^{38}$. Isoforms of AMPK are activated according to the intensity of exercise: low-intensity exercise preferentially activates the $\alpha 1$ isoform while moderate intensity exercise preferentially activates the $\alpha 2$ isoform ${ }^{11}$. In obesity and insulin resistance models, endurance training (treadmill running) increased the activity of AMPK $\alpha 1$ but not the $\alpha 2$ isoform ${ }^{39,} 40$. The mediating role of caffeine (1,3,7-trimethylxanthine) on skeletal muscle metabolism is also achieved through AMPK; low concentrations $(1 \mathrm{mM})$ of caffeine predominantly activate AMPK $\alpha 1$ via an energy-independent manner while AMPK $\alpha 2$ was activated at high concentrations $(3 \mathrm{mM})$ of caffeine, depending on energy depletion ${ }^{10}$. The polyphenols in red raspberries, such as anthocyanins, activate AMPK $\alpha 1^{41}$, consistent with our observation in this study that dietary raspberry did not significantly activate AMPK $\alpha 2$ in the skeletal muscle of obese mice.

In conclusion, we found that raspberry supplementation reduced lipid accumulation, alleviated the inflammatory response, improved insulin sensitivity, and promoted mitochondrial biogenesis in the skeletal muscle of HFD-fed mice. These beneficial effects depended on the indispensable mediator: AMPK $\alpha 1$. Further studies should focus on the signaling mechanisms of raspberry with regards to the isoform-specific activation of AMPK, which could provide new insights for the development of dietary treatments for reducing obesity and diabetes.

\section{Acknowledgements}

This study was supported by grants from the National Institutes of Health (R01HD067449 and R21-AG049976) and a grant from the National Processed Raspberry Council to M.D.

\section{Author details}

'Department of Animal Sciences, Nutrigenomics and Growth Biology laboratory, Washington State University, Pullman, WA 99164, USA. ${ }^{2}$ Jiangxi Province Key Laboratory of Animal Nutrition, College of Animal Science and Technology, Jiangxi Agricultural University, Nanchang, Jiangxi 330045, China. ${ }^{3}$ School of Food Sciences, Washington State University, Pullman, WA 99164, USA. ${ }^{4}$ Beijing Advanced Innovation Center for Food Nutrition and Human Health, College of Food Science \& Nutritional Engineering, China Agricultural University, Beijing 100194, China

\section{AUTHOR CONTRIBUTIONS}

L.Z. and M.D. designed the study and wrote the manuscript. L.Z. and T.Z. performed the experiments. L.Z., B.W., M.D. analyzed and interpreted the data. N.A.G, M-J.Z. and M.D. revised the manuscript.

\section{Conflict of interest}

The authors declare that they have no conflict of interest.

Received: 15 March 2018 Accepted: 23 May 2018

Published online: 02 July 2018

\section{References}

1. Mullen, W. et al. Ellagitannins, flavonoids, and other phenolics in red raspberries and their contribution to antioxidant capacity and vasorelaxation properties. J. Agric. Food Chem. 50, 5191-5196 (2002). 
2. Burton-Freeman, B. M., Sandhu, A. K. \& Edirisinghe, I. Red raspberries and their bioactive polyphenols: cardiometabolic and neuronal health links. Adv. Nutr. Int. Rev. J. 7, 44-65 (2016).

3. Luo, T., Miranda-Garcia, O., Adamson, A., Sasaki, G. \& Shay, N. Development of obesity is reduced in high-fat fed mice fed whole raspberries, raspberry juice concentrate, and a combination of the raspberry phytochemicals ellagic acid and raspberry ketone. J. Berry Res. 6, 213-223 (2016).

4. Petersen, K. F. \& Shulman, G. I. Pathogenesis of skeletal muscle insulin resistance in type 2 diabetes mellitus. Am. J. Cardiol. 90, 11-18 (2002).

5. Hardie, D. G. \& Sakamoto, K. AMPK: A key sensor of fuel and energy status in skeletal muscle. Physiology 21, 48-60 (2006).

6. O'Neill, H. M., Holloway, G. P. \& Steinberg, G. R. AMPK regulation of fatty acid metabolism and mitochondrial biogenesis: implications for obesity. Mol. Cell. Endocrinol. 366, 135-151 (2013).

7. Wang, S. et al. Resveratrol induces brown-like adipocyte formation in white fat through activation of AMP-activated protein kinase (AMPK) a1. Int. J. Obes. 39, 967-976 (2015).

8. Salt, I. et al. AMP-activated protein kinase: greater AMP dependence, and preferential nuclear localization, of complexes containing the alpha2 isoform. Biochem. J. 334, 177-187 (1998).

9. Woods, A., Salt, I., Scott, J., Hardie, D. G. \& Carling, D. The a1 and a2 isoforms of the AMP-activated protein kinase have similar activities in rat liver but exhibit differences in substrate specificity in vitro. FEBS Lett. 397, 347-351 (1996).

10. Egawa, T. et al. Caffeine activates preferentially a1-isoform of 5' AMP-activated protein kinase in rat skeletal muscle. Acta Physiol. 201, 227-238 (2011).

11. Kjøbsted, R. et al. Intact regulation of the AMPK signaling network in response to exercise and insulin in skeletal muscle of male patients with type 2 diabetes: illumination of AMPK activation in recovery from exercise. Diabetes $\mathbf{6 5}$, 1219-1230 (2016)

12. Jørgensen, S. B. et al. The a2-5' AMP-activated protein kinase is a site 2 glycogen synthase kinase in skeletal muscle and is responsive to glucose loading. Diabetes 53, 3074-3081 (2004).

13. Mu, J., Brozinick, J. T., Valladares, O., Bucan, M. \& Birnbaum, M. J. A Role for AMPActivated Protein Kinase in Contraction- and Hypoxia-Regulated Glucose Transport in Skeletal Muscle. Mol. Cell 7, 1085-1094 (2001).

14. Jensen, T. E., Schjerling, P., Viollet, B., Wojtaszewski, J. F. P. \& Richter, E. A AMPK a1 activation is required for stimulation of glucose uptake by twitch contraction, but not by h202, in mouse skeletal muscle. PLOS ONE 3, e2102 (2008).

15. Fu X. et al. Obesity impairs skeletal muscle regeneration via inhibition of AMPactivated protein kinase. Diabetes 65, 188-200 (2015).

16. Mounier, R. et al. AMPKa1 regulates macrophage skewing at the time of resolution of inflammation during skeletal muscle regeneration. Cell. Metab. 18, 251-264 (2013).

17. Hayashi, S. \& McMahon, A. P. Efficient recombination in diverse tissues by a tamoxifen-inducible form of cre: a tool for temporally regulated gene activation/inactivation in the mouse. Dev. Biol. 244, 305-318 (2002).

18. Zhu, M.J. et al. Dietary raspberries ameliorate metabolic syndromes in dietinduced obese mice. FASEB J. 30, 907-21 (2016).

19. Bibi, S., Kang, Y., Du, M. \& Zhu, M. J. Dietary red raspberries attenuate dextran sulfate sodium-induced acute colitis. J. Nutr. Biochem. 51, 40-46 (2018).

20. Yan, X. et al. Maternal obesity-impaired insulin signaling in sheep and induced lipid accumulation and fibrosis in skeletal muscle of offspring. Biol. Reprod. $\mathbf{8 5}$, 172-178 (2011).
21. Frayn, K. N. \& Maycock, P. F. Skeletal muscle triacylglycerol in the rat: methods for sampling and measurement, and studies of biological variability. J. Lipid Res. 21, 139-144 (1980).

22. Livak, K. J. \& Schmittgen, T. D. Analysis of relative gene expression data using real-time quantitative PCR and the $2-\triangle \triangle C T$ method. Methods 25, 402-408 (2001).

23. Zou, T. et al. Raspberry promotes brown and beige adipocyte development in mice fed high-fat diet through activation of AMP-activated protein kinase (AMPK) a1. J. Nutr. Biochem. 55, 157-164 (2018)

24. Hulver, M. W. et al. Skeletal muscle lipid metabolism with obesity. Am. J. Physiol.-Endocrinol. Metab. 284, 741-747 (2003).

25. Muoio, D. M. Intramuscular triacylglycerol and insulin resistance: guilty as charged or wrongly accused? Biochim. Biophys. Acta 1801, 281-288 (2010).

26. Hirosumi, J. et al. A central role for JNK in obesity and insulin resistance. Nature 420, 333-336 (2002).

27. Stylianou, E. \& Saklatvala, J. Interleukin-1. Int. J. Biochem. Cell. Biol. 30, 1075-1079 (1998).

28. Ye, J. \& Keller, J. N. Regulation of energy metabolism by inflammation: a feedback response in obesity and calorie restriction. Aging 2, 361-368 (2010).

29. Olson A. L. Regulation of GLUT4 and insulin-dependent glucose flux. ISRN Mol. Biol. 2012, 856987 (2012).

30. Mingrone, G. et al. Skeletal muscle triglycerides lowering is associated with net improvement of insulin sensitivity, TNF-a reduction and GLUT4 expression enhancement. Int. J. Obes. \& Relat. Metab. Disord. 26, 1165-1172 (2002).

31. Hesselink, M. K., Schrauwen-Hinderling, V. \& Schrauwen, P. Skeletal muscle mitochondria as a target to prevent or treat type 2 diabetes mellitus. Nat. Rev. Endocrinol. 12, 633-645 (2016).

32. McArdle, M. A., Finucane, O. M., Connaughton, R. M., McMorrow, A. M. \& Roche, H. M. Mechanisms of obesity-induced inflammation and insulin resistance: insights into the emerging role of nutritional strategies. Front. Endocrinol. 4, 52 (2013).

33. Witkamp, R. F. Current and future drug targets in weight management. Pharm Res. 28, 1792-1818 (2011).

34. Xing T. et al. Raspberry supplementation improves insulin signaling and promotes brown-like adipocyte development in white adipose tissue of obese mice. Mol. Nutr. Food Res. e-pub ahead of print 9 February 2018; DOl: 10.1002/ mnfr.201701035 (2018).

35. Jean-Gilles, D. et al. Anti-inflammatory effects of polyphenolic-enriched red raspberry extract in an antigen-induced arthritis rat model. J. Agric. Food Chem. 60, 5755-5762 (2011).

36. Zhang, B. B., Zhou, G. \& Li, C. AMPK: an emerging drug target for diabetes and the metabolic syndrome. Cell. Metab. 9, 407-416 (2009).

37. Stapleton, D. et al. Mammalian AMP-activated protein kinase subfamily. J. Biol. Chem. 271, 611-614 (1996).

38. Stein, S. C., Woods, A., Jones, N. A., Davison, M. D. \& Carling, D. The regulation of AMP-activated protein kinase by phosphorylation. Biochem. J. 345, 437-443 (2000).

39. Lessard, S. J. et al. Tissue-specific effects of rosiglitazone and exercise in the treatment of lipid-induced insulin resistance. Diabetes 56, 1856-1864 (2007).

40. Sriwijitkamol, A. et al. LKB1-AMPK signaling in muscle from obese insulinresistant Zucker rats and effects of training. Am. J. Physiol. Endocrinol. Metab. 290, 925-932 (2006).

41. Lee, Y.-K., Lee, W. S., Kim, G. S. \& Park, O. J. Anthocyanins are novel AMPKa1 stimulators that suppress tumor growth by inhibiting mTOR phosphorylation. Oncol. Rep. 24, 1471-1477 (2010). 